\title{
Physical mechanism of anisotropic sensitivity in pentaerythritol tetranitrate from compressive-shear reaction dynamics simulations
}

\author{
Sergey V. Zybin, ${ }^{1}$ William A. Goddard III, ${ }^{1, a)}$ Peng Xu, ${ }^{1}$ Adri C. T. van Duin, ${ }^{2}$ and \\ Aidan P. Thompson ${ }^{3}$ \\ ${ }^{1}$ Materials and Process Simulation Center, 139-74, California Institute of Technology, Pasadena, \\ California 91125, USA \\ ${ }^{2}$ Department of Mechanical and Nuclear Engineering, Pennsylvania State University, University Park, \\ Pennsylvania 16802, USA \\ ${ }^{3}$ Sandia National Laboratories, MS 1322, P.O. Box 5800, Albuquerque, New Mexico 87185, USA
}

(Received 3 November 2009; accepted 23 January 2010; published online 26 February 2010)

\begin{abstract}
We propose computational protocol (compressive shear reactive dynamics) utilizing the ReaxFF reactive force field to study chemical initiation under combined shear and compressive load. We apply it to predict the anisotropic initiation sensitivity observed experimentally for shocked pentaerythritol tetranitrate single crystals. For crystal directions known to be sensitive we find large stress overshoots and fast temperature increase that result in early bond-breaking processes whereas insensitive directions exhibit small stress overshoot, lower temperature increase, and little bond dissociation. These simulations confirm the model of steric hindrance to shear and capture the thermochemical processes dominating the phenomena of shear-induced chemical initiation. (C) 2010 American Institute of Physics. [doi:10.1063/1.3323103]
\end{abstract}

Experiments by Bridgman ${ }^{1}$ demonstrated that chemical transformations occur far more readily under combined shear and pressure loads. Coupling between mechanical, thermal, and chemical effects is important for initiation of detonation in explosives under mechanical impact. Understanding how the detonation sensitivity depends on formulation and structural properties is a critical issue in explosive technology. A great deal of effort has gone into the development of experimental techniques to study these processes (e.g., time resolved emission spectroscopy ${ }^{2-4}$ ) but they have not yet provided satisfactory resolution of initial steps of detonation. Little is known about how mechanical and chemical processes couple to initiate detonation.

An ideal system to examine for hints on the atomistic origin of sensitivity, is single crystal of pentaerythritol tetranitrate (PETN, four $\mathrm{CH}_{2}-\mathrm{O}-\mathrm{NO}_{2}$ chains connected to a central carbon, see inset of Fig. 1). Dick et al. ${ }^{7-9}$ showed that shock compression of the single crystal in different directions leads to dramatic differences in the sensitivity. Thus the pressure threshold for detonation along the [100] direction is at least $\sim 4$ times that for [110] direction. ${ }^{10}$ Such single crystal experiments eliminate many of the variables that complicate interpretations (grain boundaries, voids, and internal defects) providing an unambiguous challenge to any proposed mechanisms. Dick et al. ${ }^{8,9}$ attributed the orientational anisotropy of PETN sensitivity to steric hindrance to the shear of molecules in the neighboring slip planes. Recently, Plaksin ${ }^{11}$ showed clear-cut experimental evidence that initiation of detonation occurs preferentially in directions with the maximum shear stress.

Here we use reactive dynamics (RD) simulations at constant shear rate on uniaxially compressed PETN single crystal to show that the physical and chemical responses depend dramatically on the compression direction and slip system. In contrast, RD simulations of pure uniaxial shock compression

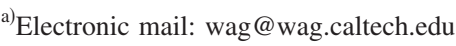

in various directions show no correlation of the reactive behavior with anisotropy of sensitivity in experiment. Key to our simulations is the ReaxFF reactive force field, ${ }^{12,13}$ whose parameters are trained to match quantum mechanics descriptions of reaction barriers for all plausible chemical bond breaking processes. But with ReaxFF it is practical to describe chemical reactions occurring at extreme shock conditions during the large scale dynamical processes involving millions of atoms. ${ }^{14}$ ReaxFF RD simulations of thermal and shock-induced decompositions have been reported on RDX, ${ }^{15-18}$ TATP ${ }^{19}$ HMX, and TATB. ${ }^{20}$ These studies provided useful data on the initial chemical decomposition processes and the subsequent-energy release processes.

Given the hint about the importance of shear, we formulated the compressive shear reactive dynamics (CS-RD) computational protocol in which the system is first uniaxially precompressed and then sheared at a constant rate along various target slip planes and slip directions. This allows us to examine each combination of compression direction and slip system independently.

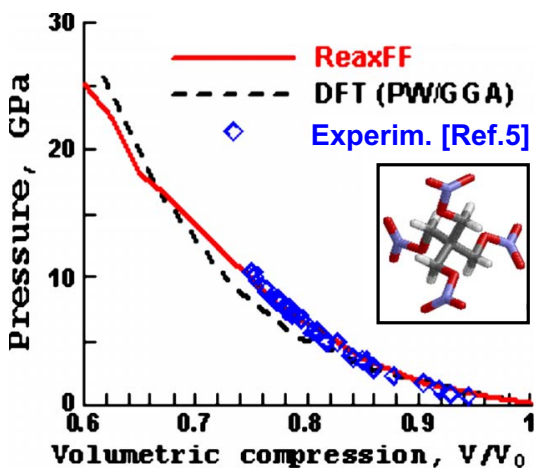

FIG. 1. (Color online) Validation of the accuracy of ReaxFF for hydrostatic compression of PETN single crystal. Experimental data (diamonds) (Ref. 5), ReaxFF (solid red line), and DFT/PBE (black dashed line) (Ref. 6). 


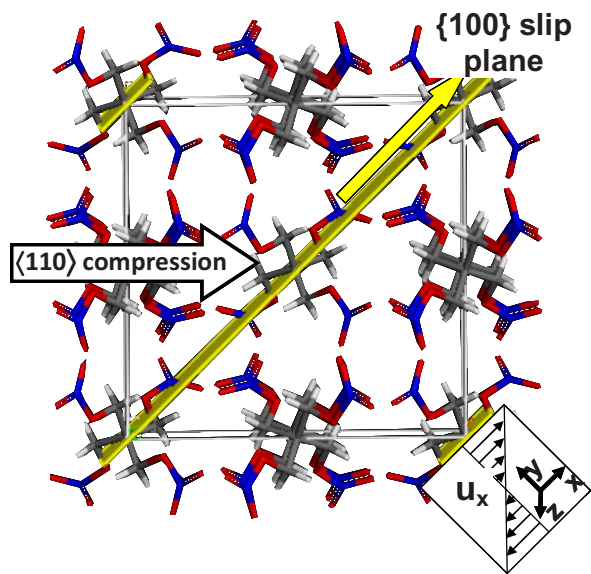

FIG. 2. (Color online) CS-RD setup for PETN single crystal. Shown is the case of $10 \%$-precompression along the $\langle 110\rangle$ direction with constant rate shear in the $\langle 011\rangle$ slip direction on the $\{100\}$ slip plane (parallel to $x z$-plane).

CS-RD uses a periodic (infinite) PETN supercell (10 $\times 10 \times 2$ in the $\mathrm{a}, \mathrm{b}$, and c directions; containing 784 molecules and 22736 atoms for the [100] shocks). This supercell was compressed by $10 \%$ along one of the experimental shock directions and then the cell was reoriented to put the slip plane parallel to a new $x z$-plane. This system was equilibrated at $T=300 \mathrm{~K}$ for one picosecond, and then sheared at a constant rate of $0.5 / \mathrm{ps}$ along the slip direction in the $x z$-plane, using nonequilibrium RD (NERD) in which the periodic supercell parameters were changed at each timestep $(0.1 \mathrm{fs})$ with atom fractional coordinates rescaled (pure shear with planar Couette flow). The applied shear acts in the $x y$-plane and changes linearly in the $y$-direction, as shown in the inset in Fig. 2.

Each shock direction leads to several possible slip systems, each of which can lead to substantial differences in the shear stress because of steric effects (in contrast to metals). The most favorable slip systems for activation are expected to be with larger resolved shear stress (RSS), lower critical resolved shear stress, and smaller Burgers vectors. The dominant cleavage plane for PETN observed in indentation experiments $^{21}$ is $\{110\}$, which Dick ${ }^{7}$ deduced to be the preferred slip system based on his estimate from the elastic constants of PETN to have the maximum RSS (MRSS) for small strains. For [110] shocks the slip system with MRSS is $\{110\}\langle 011\rangle$, while for [100] shock it is $\{110\}\langle 1 \overline{1} 1\rangle$. To avoid uncertainties in this linear elasticity approximation, we calculated the resolved shear stresses after 10\% compression along several shock directions for 11 different slip systems and then selected six MRSS systems for CS-RD.

Figure 3 shows CS-RD results at the shear rate of $0.5 / \mathrm{ps}$ for two extreme cases of sensitivity: $\langle 110\rangle$ compression (most sensitive shock direction in Dick's experiment) with shear applied on the $\{100\}\langle 011\rangle$ slip system, and $\langle 100\rangle$ compression (most insensitive) with the $\{110\}\langle 1 \overline{1} 1\rangle$ slip system. We see three stages. The first 3 ps leads only to physical interactions between molecules, with no chemistry. Here intermolecular contacts between the uniformly slipping planes causes a fast increase of shear stress $\tau_{x y}$ and normal stress $\sigma_{y y}$, that do work on the system, increasing the energy and temperature. The molecules are most jammed together at the maximum of shear stress, and then relax back as the planes slide further, leading to a jump in the temperature and an additional rise of the normal stress. The differences between the sensitive and insensitive cases are dramatic, demonstrating that the close intermolecular contacts of the sensitive direction lead to a considerably larger jumps in stress and temperature.

After $\sim 3$ ps (second stage), the first chemical reactions are observed to produce molecule fragments as follows: dissociation of $\mathrm{NO}_{2}$ from PETN, which is suggested to be the primary initiation step in PETN decomposition. ${ }^{22} \mathrm{We}$ observed an alternative initial step via $\mathrm{NO}_{3}$ reduction, but it is much less frequent, in agreement with density functional theory (DFT) based tight-binding simulations. ${ }^{23}$ During this stage, dissociation of PETN molecules leads to local disorder with other properties changing smoothly, until amorphization (third stage) starts at $\sim 6 \mathrm{ps}$ leading toward saturation of the shear stress to a constant value. The substantial decomposition and secondary products formation is observed at 20 ps. Here bimolecular and more complicated processes start playing a role.

The results for all five shock directions are shown in Table I. Here we see a perfect correlation between the stress overshoot, $\tau_{\mathrm{m}}-\tau_{\mathrm{c}}$, and the observed sensitivity. For the shocks perpendicular to (001) and to (101) there is more than one possible slip system, where $\tau_{\mathrm{m}}-\tau_{0}$ gives an estimate of the shear stress barrier to be overcome during the shear flow. For example, the $\{110\}\langle 011\rangle$ slip system for (101) shock has the largest barrier for (101) shock at $10 \%$ compression, which makes it the least preferable slip direction.

To demonstrate the role of shear on initiation of chemical processes, we also carried out NERD simulations for uniaxial shock reaction dynamics (US-RD) in PETN. In this case we moved a planar potential wall at constant piston velocity toward the PETN slab: $16 \times 4 \times 4$ supercell $(\sim 152$
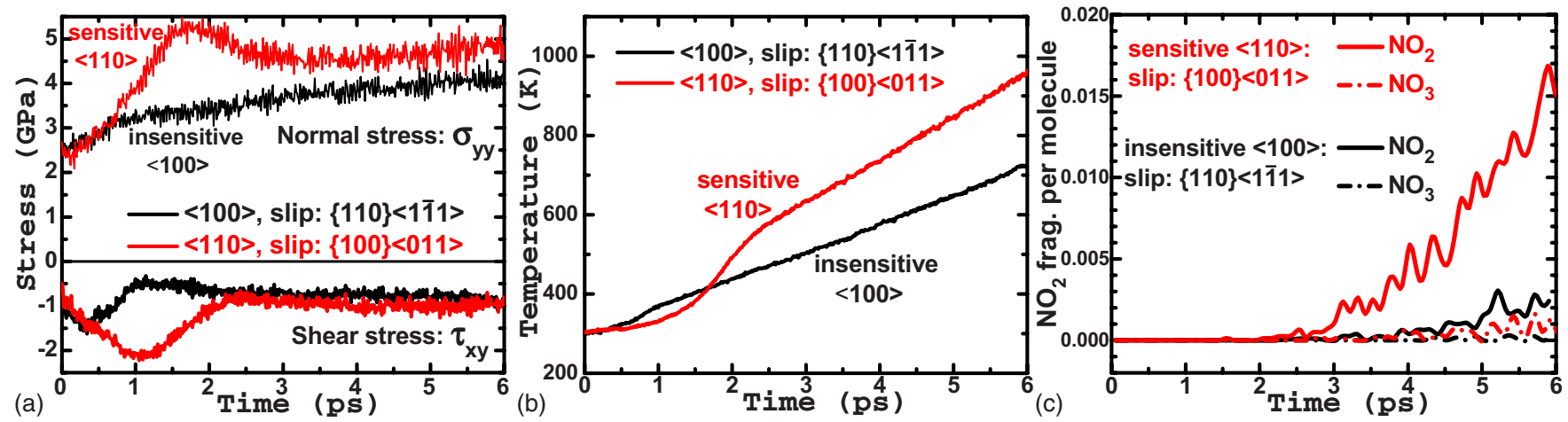

FIG. 3. (Color online) Time evolution of various processes during CS-RD for the most sensitive $\langle 110\rangle$ and most insensitive $\langle 100\rangle$ cases. (a) Shear stress $\tau_{x y}$ and normal stress $\sigma_{y y}$, (b) temperature, and (c) number of initial dissociation products $\mathrm{NO}_{2}$ and $\mathrm{NO}_{3}$ per PETN molecule. 
TABLE I. Results of CS-RD simulation (10\% precompression, $\mathrm{V}=0.9 \quad \mathrm{~V}_{0}$ ) after 6 ps. Here $\tau_{0}$ is the initial, $\tau_{\mathrm{m}}$ is the maximum, and $\tau_{\mathrm{c}}$ is the steady state shear stress. $\tau_{\mathrm{m}}-\tau_{\mathrm{c}}$ is expected to correlate with sensitivity. For some directions there is more than one choice for the slip direction. We put in bold face the one our calculations suggest to dominate (based on $\tau_{\mathrm{m}}-\tau_{0}$ ).

\begin{tabular}{ccccccc}
\hline \hline $\begin{array}{c}\text { Shock } \\
\text { plane }^{\mathrm{a}}\end{array}$ & Rank $^{\mathrm{b}}$ & $\begin{array}{c}\text { Slip } \\
\text { system }\end{array}$ & $\begin{array}{c}\mathrm{NO}_{2} \text { per } \\
\text { PETN }(\%)\end{array}$ & $\begin{array}{c}\tau_{\mathrm{m}}-\tau_{\mathrm{c}} \\
(\mathrm{GPa})\end{array}$ & $\begin{array}{c}\tau_{\mathrm{m}}-\tau_{0} \\
(\mathrm{GPa})\end{array}$ & $\begin{array}{c}\mathrm{T}(\mathrm{K}) \\
\text { at } 6 \mathrm{ps}\end{array}$ \\
\hline $\mathbf{( 1 1 0 )}$ & $\mathbf{S}$ & $\{\mathbf{1 0 0}\}\langle\mathbf{0 1 1}\rangle$ & $\mathbf{1 . 5}$ & $\mathbf{1 . 1 9}$ & $\mathbf{1 . 6 6}$ & $\mathbf{9 6 0}$ \\
$(001)$ & & $\{101\}\langle\overline{1} 01\rangle$ & 1.5 & 1.38 & 2.28 & 910 \\
$(\mathbf{0 0 1})$ & $\mathbf{S}$ & $\{\mathbf{1 0 1}\}\langle\overline{\mathbf{1 1 1}}\rangle$ & $\mathbf{0 . 8}$ & $\mathbf{1 . 0 3}$ & $\mathbf{1 . 9 3}$ & $\mathbf{8 2 6}$ \\
$(\mathbf{1 1 1})$ & $\mathbf{M}$ & $\{\mathbf{1 1 0}\}\langle\mathbf{0 0 1}\rangle$ & $\mathbf{1 . 0}$ & $\mathbf{0 . 7 6}$ & $\mathbf{1 . 0 2}$ & $\mathbf{8 3 0}$ \\
$(101)$ & & $\{100\}\langle 001\rangle$ & 2.6 & 1.60 & 1.77 & 1000 \\
$(101)$ & & $\{110\}\langle 001\rangle$ & 0.75 & 1.08 & 1.76 & 820 \\
$(\mathbf{1 0 1})$ & $\mathbf{I}, \mathbf{M}$ & $\{\mathbf{1 1 0}\}\langle\mathbf{1 1} 1\rangle$ & $\mathbf{0 . 2}$ & $\mathbf{0 . 4 5}$ & $\mathbf{1 . 0 4}$ & $\mathbf{6 9 0}$ \\
$(\mathbf{1 0 0})$ & $\mathbf{I}$ & $\{\mathbf{1 1 0}\}\langle\mathbf{1 1} 1\rangle$ & $\mathbf{0 . 2}$ & $\mathbf{0 . 4 9}$ & $\mathbf{0 . 6 9}$ & $\mathbf{7 2 5}$ \\
\hline \hline
\end{tabular}

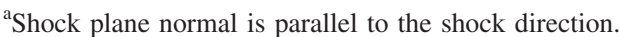

${ }^{\mathrm{b}}$ Sensitivity rank according to experiment (Refs. 7-9): S - sensitive, I insensitive, $\mathrm{M}$ - intermediate, and velocity-dependent.

$\left.\times 38 \times 28 \AA^{3}\right)$ for the [100] direction and $12 \times 3 \times 4$ supercell $\left(\sim 161 \times 40 \times 28 \AA^{3}\right)$ in the [110] direction. Because of the very small cross-section (30 to $40 \mathrm{~A}$ ), the periodic boundary conditions prevent formation of shear bands or dislocations in the shocked region. This results in purely uniaxially compressed material without shear flow.

For this US-RD we observed a significant increase in the number of dissociated $\mathrm{NO}_{2}$ and $\mathrm{HONO}$ radicals as well as other species in simulations with piston velocity $u_{p}$ $=3 \mathrm{~km} / \mathrm{s}$, where the shock velocity $\mathrm{u}_{\mathrm{s}} \sim 8.0 \mathrm{~km} / \mathrm{s}$ approaches the detonation threshold of PETN single crystal. However, Fig. 4 shows that for US-RD there is no significant difference between the sensitive [100] and insensitive [110] case, indicating that pure compression does not account for the sensitivity differences for PETN.

Summarizing, we developed and applied the CS-RD protocol to predict the directional anisotropy of chemical initiation and sensitivity of shocked single crystal explosives. We find dramatic differences in mechanical and chemical re-

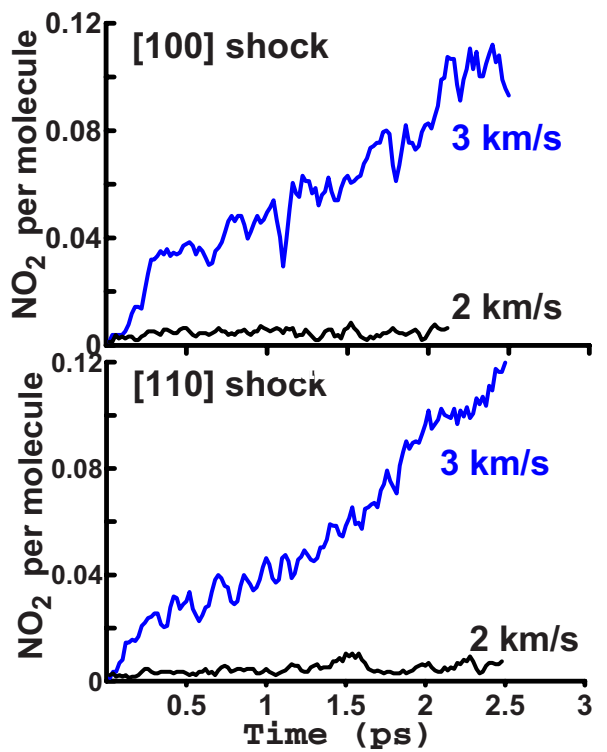

FIG. 4. (Color online) Uniaxial shock of PETN with no shear leads to very similar chemistry for both the sensitive $\langle 110\rangle$ and insensitive $\langle 100\rangle$ directions. The shock wave reaches an open surface of the crystal in $\sim 2$ ps. sponse of PETN depending on slip direction that are in agreement with shock sensitivity experiments. In particular, the shear stress overshoot $\left(\tau_{\mathrm{m}}-\tau_{\mathrm{c}}\right)$ from CS-RD correlates with observed sensitivities. This overshoot (which arises from bad steric interactions of molecules) leads to greater deformation of the molecules that in turn raises the temperature more for sensitive directions (with hindrance to shear) and increases the rate of chemical bond breaking, releasing reactive intermediates that induce further decomposition, eventually driving the detonation.

Our results show that to understand shock sensitivity of explosives, it is essential to describe the coupling between mechanical shear and chemical reactions. Thus, shock simulations without shear (US-RD) led to essentially similar rates of chemical decomposition for sensitive and insensitive directions in PETN.

This work was supported by ARO (Grant No. MURIW911NF-05-1-0345) and ONR (Grant Nos. N00014-05-10778 and N00014-09-1-0634). Simulations were performed at DOD Major Shared Resource Centers under DoD/HPCMP Challenge award (ARON27203C3K).

${ }^{1}$ P. W. Bridgman, Phys. Rev. 48, 825 (1935); J. Chem. Phys. 15, 311 (1947).

${ }^{2}$ V. K. Jindal and D. D. Dlott, J. Appl. Phys. 83, 5203 (1998).

${ }^{3}$ Y. A. Gruzdkov and Y. M. Gupta, J. Phys. Chem. A 104, 11169 (2000).

${ }^{4}$ V. Bouyer, I. Darbord, P. Hervé, G. Baudin, C. Le Gallic, F. Clément, and G. Chavent, Combust. Flame 144, 139 (2006).

${ }^{5}$ B. Olinger, P. M. Halleck, and H. H. Cady, J. Chem. Phys. 62, 4480 (1975).

${ }^{6}$ The original nitramine ReaxFF (Ref. 15) has been additionally fitted to DFT/B3LYP data on PETN including cold compression for volumes down to $\varepsilon=\mathrm{V} / \mathrm{V}_{0}=0.6$ where $\mathrm{V}_{0}$ is the initial volume. Because the London dispersion interactions are poorly described by DFT, the calculated $\mathrm{V}_{0}$ is $\sim 7 \%$ too large.

${ }^{7}$ J. J. Dick, Appl. Phys. Lett. 44, 859 (1984).

${ }^{8}$ J. J. Dick, R. N. Mulford, W. J. Spencer, D. R. Pettit, E. Garcia, and D. C. Shaw, J. Appl. Phys. 70, 3572 (1991).

${ }^{9}$ J. J. Dick and J. P. Ritchie, J. Appl. Phys. 76, 2726 (1994).

${ }^{10}$ C. S. Yoo, N. C. Holmes, P. C. Souers, C. J. Wu, F. H. Ree, and J. J. Dick, J. Appl. Phys. 88, 70 (2000).

${ }^{11}$ I. Plaskin, C. S. Coffey, R. Mendes, J. Ribeiro, J. Campos, and J. Direito, 13th Symposium (International) on Detonation, ONR 351-07-01, 2006, p. 319.

${ }^{12}$ A. C. T. van Duin, S. Dasgupta, F. Lorant, and W. A. Goddard, J. Phys Chem. A 105, 9396 (2001).

${ }^{13}$ A. C. T. van Duin, A. Strachan, S. Stewman, Q. S. Zhang, X. Xu, and W. A. Goddard, J. Phys. Chem. A 107, 3803 (2003).

${ }^{14}$ A. Nakano, R. K. Kalia, K. Nomura, A. Sharma, P. Vashishta, F. Shimojo, A. C. T. van Duin, W. A. Goddard, R. Biswas, and D. Srivastava, Comput. Mater. Sci. 48, 642 (2006).

${ }^{15}$ A. Strachan, A. C. T. van Duin, D. Chakraborty, S. Dasgupta, and W. A. Goddard, Phys. Rev. Lett. 91, 098301 (2003).

${ }^{16}$ A. Strachan, E. Kober, A. C. T. van Duin, J. Oxgaard, and W. A. Goddard, J. Chem. Phys. 122, 054502 (2005).

${ }^{17}$ K. Nomura, R. K. Kalia, A. Nakano, P. Vashishta, A. C. T. van Duin, and W. A. Goddard, Phys. Rev. Lett. 99, 148303 (2007).

${ }^{18}$ K. Nomura, R. K. Kalia, A. Nakano, and P. Vashishta, Appl. Phys. Lett. 91, 183109 (2007).

${ }^{19}$ A. C. T. van Duin, Y. Zeiri, Y. F. Dubnikova, R. Kosloff, and W. A. Goddard, J. Am. Chem. Soc. 127, 11053 (2005).

${ }^{20}$ L. Zhang, S. V. Zybin, A. C. T. Van Duin, S. Dasgupta, W. A. Goddard, and E. M. Kober, J. Phys. Chem. A 113, 10619 (2009).

${ }^{21}$ H. G. Gallagher, P. J. Halfpenny, J. C. Miller, and J. N. Sherwood, Philos. Trans. R. Soc. London, Ser. A 339, 293 (1992).

${ }^{22}$ W. L. Ng, J. E. Field, and H. M. Hauser, J. Appl. Phys. 59, 3945 (1986).

${ }^{23}$ C. J. Wu, M. R. Manaa, and L. E. Fried, MRS Symposia Proceedings Vol 987 (Materials Research Society, Pittsburgh, 2007), p. 987-PP04-11. 